\title{
Psicopatía infanto-juvenil: avances en conceptualización, evaluación e intervención
}

\section{Child and juvenile psychopathy: advances in conceptualization, assessment and intervention}

\author{
Eugenia V. Vinet \\ Universidad de La Frontera, Chile \\ (Rec: 08 julio 2009 / Acep: 20 de abril 2010)
}

\begin{abstract}
Resumen
Considerando las necesidades actuales presentes en el trabajo psicológico con adolescentes con problemas de adaptación social, este artículo realiza una revisión teórica actualizada sobre el constructo de psicopatía infanto-juvenil. Se abordan aspectos relacionados con la definición y extensión del constructo, los instrumentos de evaluación disponibles, los factores de riesgo y las perspectivas de tratamiento y prevención. En la discusión se ponderan las diferentes aproximaciones conceptuales, se plantea la conveniencia de desarrollar tanto instrumentos comprehensivos como de evaluación específica, ajustados a nuestro contexto sociocultural, y se destaca la necesidad de iniciar estudios sobre intervención que consideren las características de personalidad que están involucradas en el constructo.

Palabras Clave: Psicopatía infanto-juvenil, desadaptación social, adolescentes, delincuencia
\end{abstract}

\begin{abstract}
Considering the current needs present in the psychological approach to adolescents with social adjustment problems, this article presents an actualized theoretical review about the construct of child and juvenile psychopathy. The topics included in the revision are the construct definition and extension, the tools for assessment that are available, the risk factors and different approaches to intervention. The discussion weights the different approaches to conceptualization, it points the convenience for studying general and specific assessments tools adjusted to our sociocultural context, and it remarks the need for studying interventions that include the personality characteristics involved in the psychopathy construct.
\end{abstract}

Key words: Child and juvenile psychopathy, social maladjustment, adolescents, delinquency

\footnotetext{
Este artículo recibió financiamiento de Fondo Nacional de Desarrollo Científico y Tecnológico (FONDECYT) a través del Proyecto No 1070397 , titulado "Caracterización y evaluación multidimensional de adolescentes con desadaptación social". Su autora desea agradecer los valiosos comentarios sobre su contenido realizados por la Ps. y Mg. Paula Alarcón Bañares y los generosos aportes del Ps. y Dr. José L. Saiz.

La correspondencia sobre este artículo debe dirigirse a la Dra. Eugenia V. Vinet, Departamento de Psicología, Universidad de La Frontera, Casilla 54-D, Temuco, Chile. Email: evinet@ufro.cl
} 


\section{Introducción}

En los últimos años, tanto en Chile como en otros países, ha aumentado significativamente la tasa de niños y jóvenes involucrados en conductas violentas y delitos (Alarcón, Vinet \& Salvo, 2005; Werth \& Sepúlveda, 2003). Para enfrentar esta situación se han generado diversas medidas que incluyen, en nuestro país, la promulgación de la Ley de Responsabilidad Penal Juvenil (LRPJ) en el año 2007. Esta ley señala que los adolescentes son sujetos de derecho y que como tales se les puede acusar y/o exigir una responsabilidad social especial adecuada a su carácter de sujetos en desarrollo, con sanciones distintas a las aplicadas en el adulto; además, la LRPJ da cabida a la formalización de diversos programas de intervención orientados a la prevención y tratamiento de los jóvenes con problemas de adaptación social.

En este contexto se han desarrollado numerosos estudios, teóricos y empíricos, con el fin de abordar esta problemática juvenil. En estos trabajos, el concepto de psicopatía infanto-juvenil ha sido un concepto resistido por los investigadores y profesionales que trabajan con niños y jóvenes con problemas de adaptación social. Sin embargo, en la actualidad se hace muy necesario incorporar el conocimiento que, a nivel internacional, ha generado su estudio pues éste puede ayudarnos en el abordaje y la generación de estrategias de intervención de niños y jóvenes con desadaptación social y delincuencia. Considerando esta necesidad, en este artículo se realiza una revisión de la psicopatía infanto-juvenil considerando tres aspectos fundamentales:

1. Definición y teorización ¿Qué es la psicopatía infanto-juvenil? ¿Cómo surge este concepto y como se relaciona con la psicopatía adulta?

2. Medición. ¿Cómo puede ser evaluada la psicopatía infanto-juvenil? ¿Cuáles son los instrumentos disponibles?

3. Factores de riesgo, prevención y tratamiento. ¿Cuáles son los factores de riesgo para la psicopatía en la infancia y la adolescencia? ¿Qué lugar ocupa la psicopatía infanto-juvenil como factor de riesgo para la delincuencia? ¿Hasta que punto la psicopatía en la infancia y adolescencia puede ser prevenida y tratada?

\section{Definición y conceptualización}

La psicopatía es un constructo polémico pero de gran relevancia clínica y criminológica que ha sido utilizado para designar a personas con un trastorno antisocial severo, crónico y difícil de tratar. Generalmente describe a delincuentes adultos que tienen una alta tasa de delitos, alta probabilidad de delitos violentos, alta proporción de reincidencia y mala respuesta al tratamiento (Essau, Sasagawa \& Frick, 2006; Romero, Luengo, Gómez-Fraguela, Sobral \& Villar, 2005). También es utilizado para caracterizar a personas que poseen un estilo de vida antisocial, que no necesariamente se expresará en alto número de delitos sino, más bien, en características personales que implican encanto y habilidad para manipular, engañar y usar diferentes estrategias para alcanzar sus propósitos; estas personas usarán la violencia cuando el encanto, la manipulación, y la intimidación no sean efectivos (Hare, 2003).

La conceptualización de la psicopatía surge desde de dos grandes tradiciones: una clínica y otra conductual. La tradición clínica, recogida en parte en los criterios de la CIE-10 para la Personalidad Disocial (Organización Mundial de la Salud, 1992), enfatiza la presencia de rasgos de personalidad como falta de empatía, dificultades de planificación, déficits afectivos, egocentrismo y ausencia de remordimientos. La tradición conductual pone el acento en los comportamientos trasgresores públicamente observables presentes en una historia de conducta antisocial reiterada cuyos indicadores están mejor conceptualizados en el Trastorno Antisocial de la Personalidad (TAP) descrito, con algunas variaciones, en las sucesivas versiones del DSM desarrolladas por la Asociación de Psiquiatría Americana (Romero, 2001).

En la actualidad, la conceptualización de Hare (1991, 2003 ) es la que ha generado mayor consenso. Ella incluye dos grandes factores: (a) uno referido a características personales y afectivas, está integrado por rasgos de egocentrismo, falta de sinceridad, insensibilidad y falta de remordimientos; (b) el segundo integra los aspectos conductuales a través de la evaluación de un estilo de vida desviado con conductas antisociales manifiestas. En la segunda versión de esta conceptualización, Hare (2003) propone una nueva estructuración en la cual subdivide cada uno de los dos factores originales en dos facetas más específicas; los cuatro factores que se reportan en la actualidad son: Afectivo, Interpersonal, Estilo de vida y Antisocial, y están asociados a ítems específicos del Psychopatic Checklist Revised (PCL-R), instrumento que representa esta concepción de la psicopatía adulta (Hare, 2003).

Una conceptualización alternativa es planteada por Cooke y Michie (2001). Ésta incluye tres dimensiones que abarcan: (1) lo interpersonal, a través de un estilo arrogante, engañoso y artificioso, con rasgos de locuacidad, encanto superficial, egocentrismo y manipulación; (2) lo afectivo, expresado en escaso remordimiento y culpa, crueldad, baja empatía, afectos superficiales y fracaso en aceptar la propia responsabilidad por las acciones; (3) lo impulsivo, expresado en un estilo conductual irresponsable que incluye aburrimiento, búsqueda de la estimulación, carencia de metas a largo plazo, impulsividad, ausencia de reflexión antes de actuar y un estilo de vida parasitario. Como puede observarse, esta definición no incluye, en sí misma, la conducta antisocial ni trasgresiones específicas. A juicio de Farrington (2005), esta conceptualización permitiría el estudio de la psicopatía de una forma más pura, como una posible causa de la conducta antisocial, lo que no podría 
realizarse si en la propia definición se incluyen las conductas antisociales que se intentan explicar o comprender.

\section{Raíces y evolución de la conceptualización de la psicopatía infanto-juvenil}

La psicopatía infanto-juvenil es un constructo que cada vez más reclama la atención de los investigadores en psicología del desarrollo, psicopatología y psicología jurídica como un elemento importante para caracterizar a niños y jóvenes con problemas de desadaptación social, predecir su trayectoria delincuencial y definir estrategias de intervención o tratamiento (Pardini \& Loeber, 2007). A la vez, es un concepto que produce rechazo pues tiene un halo fatalista vinculado a la inmodificabilidad de las características involucradas en su definición.

Para examinar el surgimiento de este constructo hay que recabar información a partir de la década de 1940, donde Cleckley (1941), en su monografía "The Mask of Sanity", reconocía que la psicopatía probablemente tenía sus raíces en la niñez o en la adolescencia. Un poco más tarde, Karpman $(1949,1950)$ dirigió dos mesas redondas consecutivas donde se discutió la aplicabilidad del constructo de psicopatía a los niños; en estas sesiones se discutieron muchos de los tópicos vigentes en el estudio actual del constructo como, por ejemplo, los elementos que definen la psicopatía, su etiología y posibilidad de tratamiento. Una década más tarde, McCord y McCord en su ensayo "The Psychopath: An Essay on the Criminal Mind", enfatizaban la importancia de identificar y tratar la psicopatía en población juvenil como un trastorno específico y diferente de otros problemas de conducta. Estos autores señalaban que sólo el $14 \%$ de los jóvenes con problemas de conducta cumplían con las características de un trastorno psicopático de la personalidad; además destacaban la importancia de intervenciones tempranas y de proveer un tratamiento adecuado a los jóvenes con problemas de conducta que mostraban rasgos psicopáticos (McCord \& McCord, 1959/1964, citado en Salekin \& Frick, 2005). Casi al mismo tiempo, Quay, una figura clave en la investigación sobre la clasificación de la psicopatología infantil, planteaba que una clasificación precisa de subcategorías homogéneas de jóvenes antisociales -entre las cuales estaba la psicopatía- permitiría una mejor aproximación a las alternativas de tratamiento para estos jóvenes (Salekin \& Frick, 2005). Más tarde, Quay (1986, citado en Salekin y Frick, 2005) se refiere a los jóvenes psicópatas como "infrasocializados agresivos" en un intento de proveer una etiqueta diagnóstica menos peyorativa.

\section{Diagnóstico de la psicopatía infanto-juvenil}

Para establecer el diagnóstico de psicopatía infanto juvenil la literatura se focaliza en el Trastorno de Conducta (TC), categoría diagnóstica que el DSM contempla como un requisito para el diagnóstico de TAP. El TC designa patrones de comportamiento antisocial persistentes y extremos para el nivel evolutivo del niño, que entran en conflicto con las normas y los derechos o necesidades de los demás; se trata de una categoría heterogénea que agrupa múltiples tipos de conducta, trayectorias y etiologías (Romero, 2001).

El TC, como categoría diagnóstica, ha cambiado a través de las sucesivas versiones del DSM. Así, en el DSM-III se contemplaban cuatro tipos que resultaban de la combinación de dos criterios: socialización y agresión; el tipo Infrasocializado Agresivo, propuesto por Quay (1986, citado en Salekin y Frick, 2005) recoge implícitamente algunas de las características de la psicopatía al caracterizar a niños con dificultades para establecer vínculos afectivos, escasa capacidad para empatizar, egocentrismo, falta de remordimiento, conducta agresiva y arriesgada; a diferencia de éste, el tipo Socializado Agresivo agruparía a niños que desarrollan conductas antisociales en pandillas, como parte de una subcultura en la que existen importantes vínculos grupales; estos niños y jóvenes no ameritarían el diagnóstico de psicópatas.

En el DSM-III-R, debido a dificultades para clasificar objetivamente según lo interpersonal-afectivo, los subtipos del TC se definen en función de si los actos antisociales se cometen en grupo o en solitario; puesto que los antisociales "solitarios" tendían a tener altos niveles de agresividad se retienen dos tipos: el Solitario Agresivo y el Grupal. Así, de modo similar a lo ocurrido en el TAP, se van eliminando las referencias a características personales o rasgos, más difíciles de objetivar, privilegiando descripciones más conductuales (Romero, 2001).

En el DSM-IV, el criterio para distinguir subgrupos de niños con TC es la edad de inicio, distinguiéndose un tipo de inicio infantil y un tipo de inicio adolescente; esta distinción esta avalada por el trabajo de Moffitt (1993) que revisa e integra la información obtenida hasta esa fecha y propone un modelo sobre la conducta antisocial juvenil. De acuerdo a Moffitt (1993), los niños en los cuales la conducta problemática aparece temprano tienen una trayectoria evolutiva más persistente y mayor riesgo de consolidar una psicopatía en la adultez; por su parte, la conducta antisocial de inicio adolescente sería una forma transitoria de enfrentar las demandas madurativas de la etapa adolescente.

Esta discusión respecto del diagnóstico no está acabada pues día a día aparecen nuevos estudios que relativizan o refuerzan determinados aspectos; tal es el caso, por ejemplo, de Saltaris (2002) quien pone el énfasis en las nociones de temperamento y apego en la temprana infancia como precursores específicos de la orientación aparentemente amoral, desafectada y autocentrada que los jóvenes psicópatas despliegan en su vinculación interpersonal.

\section{Conceptualizaciones actuales}

Actualmente en la psicopatía infanto-juvenil coexisten dos aproximaciones que se acercan de modo diferente al 
TC. La primera implica un subtipo de TC basado en su comorbilidad con la hiperactividad y la impulsividad (Lynam, 1996, 1997); esta aproximación plantea que los niños con comportamiento impulsivo/hiperactivo y problemas de conducta tienden a presentar mayor riesgo de conductas antisociales severas y persistentes que aquellos niños que sólo presentan una de las dos condiciones. La segunda aproximación, más restrictiva, privilegia características específicas asociadas a los rasgos de crueldad y frialdad emocional o insensibilidad que se presentan en un subgrupo pequeño de niños con trastornos de conducta e impulsividad/hiperactividad (Frick, Barry \& Bodin, 2000, citado en Gacono, 2000). Estas características se asemejan a las descritas en la psicopatía adulta a través del componente afectivo de las conceptualizaciones de Hare (2003) y de Cooke y Michie (2001) y serían las responsables de un trastorno antisocial crónico y severo en niños y adolescentes.

Estas conceptualizaciones tienen orígenes teóricos diferentes. La de Lynam $(1996,1997)$, conocida como "la hiperactividad en el psicópata incipiente", parte de categorías diagnósticas bien identificadas - trastorno de conducta y trastorno por hiperactividad- $\mathrm{y}$ busca precisar cómo ellas se aproximan al constructo de psicopatía adulta (Romero, 2001). Lynam (1996), a partir de observaciones empíricas en las que se comparan grupos de niños con diferentes condiciones (hiperactividad solo, trastorno de conducta solo e hiperactividad+trastorno de conducta), plantea que la co-ocurrencia de estas dos condiciones permite identificar a niños que presentan patrones de conducta antisocial severa y que se caracterizan, además, por una edad de inicio más temprana, mayor frecuencia, severidad y variedad en la conducta antisocial, y manifestaciones antisociales en diferentes contextos (hogar y escuela). La hipótesis de Lynam (1996) es que este grupo especial de niños con trastorno de conducta e hiperactividad presenta un déficit de inhibición asociado al sistema serotonérgico el cual sería responsable de los síntomas propios del TDAH como la inquietud motora y la hiperactividad, la falta de atención en actividades que buscan recompensa y las conductas impulsivas y, además, sería responsable del "choque" con las normas. Este choque con las normas, asociado a los déficits de inhibición, daría lugar a conductas desafiantes tempranas que, unidas a una espiral de consecuencias adversas (endurecimiento o ambigüedad en la disciplina parental, mala adaptación en el colegio, conflictos con los pares), conducirían al trastorno de conducta con características psicopáticas. Así, según Lynam (1996), la psicopatía infanto-juvenil estaría localizada, en las taxonomías de uso común, en el subgrupo de niños que presenta conjuntamente trastorno de conducta $\mathrm{e}$ hiperactividad.

Frick y Ellis (1999) realizan dos observaciones sobre el trabajo de Lynam $(1996,1997)$. Por un lado, observan que la co-ocurrencia entre TDAH y TC es muy alta (entre el $65 \%$ y el $90 \%$ en muestras clínicas con TC) y no compatible con el número notablemente inferior de adultos diagnosticados con psicopatía (alrededor del 15\%); por otro lado, señalan que la propuesta de Lynam, basada en la combinación de pobre control de impulsos y conducta antisocial, no refleja ciertas características "críticas" de la psicopatía como la insensibilidad emocional y la falta de remordimientos (Frick \& Ellis, 1999).

El trabajo de Frick (Frick, Bodin \& Barry, 2000; Frick \& Ellis, 1999) parte directamente del concepto de psicopatía adulta e intenta comprobar si los rasgos centrales de este constructo permiten delimitar a un grupo especial de niños. Para ello desarrolla diferentes instrumentos acordes con el modelo de Hare que son sometidos a análisis factorial. Un primer estudio, realizado con el Psychopathy Screening Device o PSD (Frick, O'Brien, Wooton \& McBurnett, 1994), que utiliza como informantes a padres y profesores de 92 niños con problemas clínicos. El estudio revela una estructura de dos factores que son denominados Impulsividad/ Problemas de Conducta (I/PC) y Dureza/Insensibilidad emocional (D/I); estos dos factores son muy semejantes a los dos factores que la literatura ha identificado con la psicopatía en muestras adultas, abarcando los aspectos conductuales y los aspectos afectivo/interpersonales. Frick et al., (1994), a partir del análisis de diversas interacciones significativas entre variables, sugieren que el factor I/PC captura un constructo muy similar a las definiciones tradicionales de problemas de conducta y que valores altos en el factor D/I permiten identificar a un subgrupo de niños con problemas de conducta que tienen características especiales relacionadas con falta de sentimientos del culpa, emociones superficiales y falta de empatía. Así, este estudio parece ser el punto de partida para la investigación y sistematización, en niños y adolescentes, de la dimensión Dureza/ Insensibilidad que rescata los rasgos de crueldad y frialdad emocional que actualmente se asocian a la conceptualización de la psicopatía infanto-juvenil; esta dimensión está siendo estudiada en niños y adolescentes judicializados y de población general.

Una revisión de estudios desarrollados en muestras judicializadas (Andershed, Gustafson, Kerr \& Stattin, 2002), informa que los jóvenes que presentan las características de crueldad, indiferencia afectiva y frialdad emocional asociadas al factor D/I, cometen más delitos y de mayor gravedad que los adolescentes trasgresores no psicopáticos, comienzan sus actividades trasgresoras a más temprana edad y están más involucrados en violencia institucional; estos autores plantean que la detección de estas características personales debería realizarse antes de que los jóvenes tengan contacto con el sistema judicial o necesiten tratamiento clínico, es decir en contextos no judicializados.

En población general, la investigación ha sido más restringida y se ha centrado en cómo capturar las características de la psicopatía en jóvenes que no han sido señalados como antisociales por los sistemas judiciales pero que están en 
riesgo de conducta antisocial y delincuencia. En muestras de escolares, Essau et al., (2006) y Frick et al. (2000) señalan que los rasgos de crueldad, desafección y frialdad emocional, al combinarse con problemas de conducta, son los que mejor detectan la psicopatía en niños y jóvenes de los dos sexos. Además, estos autores señalan, de común acuerdo con Dadds, Fraser, Frost y Hawes (2005), que aunque las dimensiones que emergen en niños y jóvenes de población general no son tan claras como las que surgen en los adultos y jóvenes judicializados, las características de carencia de culpa, remordimiento, emocionalidad y empatía, junto a un uso manipulativo de los otros en función del propio beneficio, constituyen el elemento diferenciador del subgrupo psicopático en poblaciones escolares. El desafío siguiente es poder caracterizar estos aspectos asociados a la psicopatía de modo tal que pueda ser pesquisada y evaluada tempranamente.

\section{Medición de la psicopatía infanto-juvenil}

\section{Psychopathy Checklist:Youth Version.}

Una revisión de las principales herramientas para medir y evaluar la psicopatía en la niñez y adolescencia muestra que el instrumento per se, es la Psychopathy Checklist: Youth Version o PCL:YV (Forth, Kosson, \& Hare, 2003). La PCL:YV es una adaptación de la PCL-R de adultos (Hare, 2003) y busca evaluar rasgos psicopáticos en adolescentes entre 12 y 18 años. Al igual que la PCL-R consta de 20 ítems orientados a evaluar rasgos conductuales que se puntúan según su presencia o ausencia en una escala de tres puntos, utilizando la información obtenida en una exhaustiva entrevista clínica semi-estructurada e información procedente de registros externos. Se trata de un instrumento complejo que debe ser aplicado por profesionales clínicos especialmente capacitados involucrando un tiempo de aplicación cercano a dos horas. Su información, al igual que la proporcionada por PCL-R, ha sido organizada en dos grandes factores subdivididos en cuatro facetas: afectivo, interpersonal, conducta impulsiva y antisocial. A diferencia de la escala de adultos, la PCL:YV no posee un puntaje de corte clasificatorio.

Entre sus características psicométricas se señala que, luego del intenso entrenamiento requerido para su aplicación, la PCL:YV posee un índice de acuerdo inter-jueces entre .90 y .96 y una consistencia interna entre .85 y .94 (Forth et al., 2003). Roberts y Bender (2006) señalan que, en diversos estudios, se han encontrado correlaciones moderadas con informes de delincuencia, síntomas externalizados y agresión; también plantean que la PCL:YV es capaz de predecir significativamente tanto reincidencia violenta como no violenta y trasgresiones disciplinarias. Sin embargo, no todos los reportes son positivos; por ejemplo, se ha fracasado en encontrar evidencia de la relación entre la PCL:YV y el progreso en el tratamiento de jóvenes judicializados (Spain,
Douglas, Poythress, \& Epstein, 2004). A nivel nacional, es importante señalar que la PCL:YV cuenta con un estudio psicométrico preliminar realizado con 30 adolescentes varones judicializados, de entre 13 y 17 años, que señala una adecuada confiabilidad e índices positivos de validez concurrente con las escalas del Inventario Clínico para Adolescentes de Millon (MACI), Millon, 1993) que están asociadas a conducta antisocial (Zúñiga, 2009).

\section{Instrumentos alternativos}

Considerando la gran inversión en recursos humanos calificados, entrenamiento, y tiempo de aplicación que requiere la PCL-YV, se han buscado instrumentos alternativos. Entre ellos sobresale la Antisocial Process Screening Device o APSD (Frick \& Hare, 2001), la cual es una reformulación del PSD (Frick et al., 1994). La APSD busca evaluar los rasgos precursores de la psicopatía según el modelo de Hare $\mathrm{y}$, aunque está orientada preferentemente a niños entre $6 \mathrm{a}$ 13 años, es utilizada extensamente con adolescentes. Sus 20 ítems, análogos a los del PCL-R, se puntúan en una escala de tres puntos que aportan a un puntaje total y a tres dimensiones de comportamiento: insensibilidad emocional, narcisismo e impulsividad. La escala debe ser completada preferentemente por adultos próximos al niño como padres y/o profesores; sin embargo, también existe una versión autoinformada para adolescentes. Ambas versiones han mostrado buenas características psicométricas (Frick \& Hare, 2001; Muñoz \& Frick, 2007).

Desde un aproximación basada en el autorreporte del adolescente como fuente de información, se han desarrollado el Youth Psychopathic Traits Inventory o YPI (Andershed, Kerr, Stattin, \& Levander, 2002, citado en Blauuw y Sheridan, 2002), y dos escalas derivadas del Inventario Clínico para Adolescentes de Millon o MACI (Millon, 1993).

El YPI es un autoreporte de 50 ítems diseñado especialmente para evaluar psicopatía en adolescentes superando las dificultades de medir rasgos psicopáticos a través de autoreporte en los jóvenes. Consecuentemente, el YPI presenta los rasgos psicopáticos como habilidades con el objeto de minimizar problemas de distorsión de respuesta y deseabilidad social (e.g., uno de sus ítems dice: "Soy bueno para hacer que la gente crea en mi cuando yo hago algo"). El YPI está orientado a jóvenes entre 12 y 18 años y entrega puntajes para la escala total, tres dimensiones factoriales y 10 subescalas; posee estudios que avalan su idoneidad para trabajar con jóvenes de población general, con problemas judiciales y buenas relaciones con la PCL:YV (Green, 2005).

Desde una perspectiva más general, se han desarrollado dos escalas derivadas del MACI: la Escala de Contenido Psicopático o PCS (Murrie \& Cornell, 2000) y la Escala Psicopática de 16-ítems o P-16 (Salekin, Ziegler, Larrea, Anthony \& Bennett, 2003). Ellas merecen especial atención 
debido al amplio uso que el MACI está teniendo en nuestro país, especialmente en el área de la desadaptación social y delincuencia juvenil (Alarcón et al., 2005; Alarcón, Vinet, Salvo \& Perez-Luco, 2009).

La PCS (Murrie \& Cornell, 2000) está integrada por 20 ítems del MACI que se relacionan conceptualmente con el constructo bidimensional de psicopatía de Hare (1991), ha presentado buenas correlaciones con ambos factores y una capacidad de discriminación del $83 \%$ (sensibilidad $=.85$, especificidad $=.81)$ con respecto a alta y baja psicopatía clasificada según la PCL-R. La P-16 (Salekin et al., 2003) fue desarrollada conceptualmente teniendo en cuenta las propuestas de Hare $(1991,2003)$ y los planteamientos de Cooke y Michie (2001); quedó conformada por tres subescalas cuyos ítems reflejan las dimensiones de dureza/insensibilidad, egocentrismo y conducta antisocial. Su estudio inicial (Salekin et al., 2003) documenta una buena capacidad para predecir reincidencia general y reincidencia violenta como escala total y según sus facetas dureza/insensibilidad y conducta antisocial. En Chile, las dos escalas han sido utilizadas exploratoriamente $\mathrm{y}$ han resultado especialmente atractivas pues brindan una evaluación rápida y sencilla de un constructo que claramente está asociado a la trasgresión social juvenil (Alarcón et al., 2009; Zúñiga, 2009).

Un último instrumento a describir es una escala diseñada por Frick (2003) para evaluar específicamente la dimensión Dureza/Insensibilidad en adolescentes; se trata del Inventory of Callous-Unemotional Traits o ICU (Frick, 2003; Essau et al., 2006). Esta es una escala de autoreporte que consta de 24 ítems que se estructuran en tres factores: Dureza (callousness; dimensión del comportamiento que incluye carencia de empatía, culpa y remordimiento), Descuido interpersonal (uncaring; dimensión que incluye conductas relacionadas con la ausencia de cuidado por el propio desempeño y por los sentimientos de los demás) y Frialdad emocional (unemotional; focalizada en la ausencia de expresión emocional). El estudio del ICU a través de análisis factorial confirmatorio, el examen de los efectos de genero y edad, su asociación con problemas psicosociales, psicopatología y personalidad y su poder de predicción de la conducta antisocial, han contribuido a maximizar el conocimiento de la dimensión $\mathrm{D} / \mathrm{I}$ con respecto a las posibles trayectorias de desarrollo de estos rasgos, su asociación con rasgos temperamentales de la personalidad como la búsqueda de sensaciones y la carencia de inhibiciones conductuales y además, la asociación negativa de los rasgos psicopáticos con la ansiedad (Essau et al., 2006).

\section{Aportes de los instrumentos de evaluación}

El estudio de las propiedades psicométricas de los instrumentos descritos ha aportado a una mejor comprensión de la psicopatía infanto-juvenil señalando algunas diferencias con respecto a la psicopatía en adultos. Por ejemplo, se ha observado que la estructura de dos factores de Hare (1991, 2003), que refleja la psicopatía en adultos, es la más frecuente en los estudios con adolescentes judicializados; sin embargo, ella no obtiene el mismo ajuste en muestras clínicas y comunitarias donde los dos factores no aparecen con la misma claridad; además, en muestras juveniles no judicializadas los ítems que evalúan narcisismo suelen pesar de modo importante en la dimensión Impulsividad/Problemas de Conducta del APSD y no en Dureza /Insensibilidad como ocurre en muestras adultas (Frick, et al., 2000, citado en Gacono, 2000). En otro ejemplo, al comparar la validez predictiva de las dos dimensiones de Hare $(1991,2003)$ y las tres de Cooke y Michie (2001) sobre la reincidencia, se ha encontrado que el modelo de Hare es el que mejor la predice, posiblemente porque incluye la medida tautológica de conducta antisocial; de las tres dimensiones de Cooke y Michie (2001), la dimensión conductual-estilo conductual impulsivo e irresponsable- es el mejor predictor de reincidencia (Corrado, Vincent, Hart \& Cohen, 2004). Por último, es necesario destacar los diversos estudios orientados a evaluar correctamente la dimensión Dureza/Insensibilidad como predictor de la severidad y estabilidad de problemas de conducta y delincuencia y en el desarrollo de trayectorias de problemas conductuales severos.

\section{Factores de riesgo, prevención y tratamiento}

El abordaje de este apartado es triple. Por un lado, interesa analizar cuáles son los factores de riesgo para la psicopatía en la infancia y la adolescencia; por otro lado, interesa evaluar el lugar que ocupa la psicopatía infantojuvenil como factor de riesgo para la delincuencia en jóvenes y adultos; en tercer lugar, interesa describir cual es el estado del arte con respecto a prevención y tratamiento. Los aspectos que vinculan estas diferenciaciones se relacionan con los planteamientos sobre un modelo evolutivo de la psicopatía infanto-juvenil, que plantea trayectorias de desarrollo diferenciadas y abordajes de prevención y tratamiento diferenciados.

\section{Factores de riesgo para la psicopatía}

Según Farrington (2005), el énfasis histórico sobre los factores de riesgo que en la niñez temprana pueden predecir, influir o causar psicopatía ha estado centrado en los correlatos biológicos de la psicopatía adulta existiendo un énfasis menor en los factores familiares que pueden afectar a niños y jóvenes. Una revisión de factores biológicos asociados a la psicopatía, realizada por París (2003, citado en Millon, Simonsen, Birket, Smith y Davis, 2003), señala características neuropsicológicas específicas como la dificultad para desarrollar respuestas condicionadas a estímulos relacionadas con el miedo y una variación temperamental denominada "temperamento desinhibido", además destaca la comorbilidad que se establece con el TDAH y que la gran 
diferenciación de género en la prevalencia de la psicopatía podría estar relacionada a factores genéticos.

Entre los factores psicológicos se destacan los factores familiares. Autores de la década de 1950 como Bowlby y McCord y McCord señalaban tempranamente la influencia de la familia como antecedente para la psicopatía (Farrinton, 2005). De acuerdo a Bowlby, si un niño sufría un prolongado período de deprivación maternal durante los primeros cinco años de vida tendría efectos negativos irreversibles que incluían llegar a desarrollar un carácter "frío no afectuoso" y delincuencia; según McCords y McCord, el rechazo parental, un padre antisocial, la disciplina errática y la supervisión parental pobre influían en el desarrollo de la psicopatía (Farrington, 2005). Estudios más recientes refuerzan la idea del rol de la influencia parental (conducta antisocial en los padres, alcoholismo parental, fracaso parental en la disciplina y supervisión de los niños) en los orígenes de la psicopatía (Paris, 2003; en Millon, Simonsen, Birket.Smith y Davis, 2003).

La investigación actual está integrada preferentemente por estudios retrospectivos con poca presencia de estudios longitudinales. Los primeros muestran, por ejemplo, que al comparar delincuentes con puntuaciones altas y bajas en el PCL-YV, los primeros habían sufrido con mayor frecuencia abuso físico y separación de los padres que los delincuentes no psicopáticos (Campbell, Porter \& Santor, 2004); en otro ejemplo, al comparar prisioneros psicopáticos y no psicopáticos usando el PCL-R, se reporta que los primeros habían experimentado indiferencia parental o negligencia, supervisión parental pobre y disciplina parental pobre (Marshall \& Cooke, 1999); otros reportes señalan que la pertenencia a hogares desintegrados, familias uniparentales y experiencias de abuso infantil, serían factores de riesgo para el desarrollo de características psicopáticas (para una revisión ver Forth \& Burke, 1998, citado en Cooke, Forth y Hare, 1998).

Los pocos estudios longitudinales encontrados (Lang, Klinteberg \& Alm, 2002; Weiler \& Widom, 1996;) indican que el abuso infantil y la negligencia parental son factores que inciden en puntuaciones altas en la PCL-R en la adultez. Aunque estos estudios destacan los componentes familiares como factor de riesgo para la psicopatía, hay diversos autores indican que el foco correcto de estudio debería estar, no en factores aislados, sino que en la interacción entre variables biológicas y psicosociales en el seno de la familia (Farrington, 2005).

\section{Psicopatía infanto-juvenil como factor de riesgo}

La psicopatía infanto-juvenil como factor de riesgo para la delincuencia es estudiada generalmente a través de las características conductuales y los rasgos de personalidad que están incluidos en la conceptualización de psicopatía. En este contexto uno de los factores de riesgo más estudiados en muestras infantiles y juveniles ha sido la presencia de los rasgos agrupados en la dimensión Dureza /Insensibilidad de la psicopatía.

En este ámbito, destaca un estudio longitudinal del equipo de Frick (Frick, Cornell, Barry, Bodin \& Dane, 2003; Frick, Cornell, Bodin, Dane, Barry \& Loney, 2003; Frick, Kimonis, Dandreaux \& Farrell, 2003; Frick, Stickle, Dandreaux, Farell \& Kimonis, 2005) que dimensionó la presencia de problemas de conducta y de los rasgos de Dureza/ Insensibilidad en niños y adolescentes de una muestra comunitaria que fue evaluada anualmente a lo largo de cuatro años. Los hallazgos de este grupo de estudios son contundentes en varios aspectos: (1) Se encontró que los niños con rasgos de $\mathrm{D} / \mathrm{I}$ y problemas conductuales tuvieron, desde el primer seguimiento, un mayor número y mayor variedad de problemas conductuales que los niños que inicialmente sólo tenían altos niveles de problemas de conducta; estos niños también mostraron niveles más elevados de agresión, especialmente agresión instrumental, mayor autoreporte de delincuencia y, además, presentaron una mayor tasa acumulativa de contactos con la policía que los niños con problemas conductuales pero sin rasgos de D/I (Frick et al., 2005). (2) Se encontró que la estabilidad de los rasgos psicopáticos está relacionada con el contexto psicosocial del niño, específicamente con el estatus socioeconómico y la calidad de las estrategias parentales de cuidado, siendo importante el cuidado parental en el desarrollo de la culpa, la empatía y otros aspectos de la consciencia (Frick, et al., 2003). (3) Se observó que los niveles más altos de problemas conductuales en el niño se asocian con niveles más estables de rasgos psicopáticos; al respecto se plantea que es probable que el involucramiento crónico en conducta antisocial, agresiva y criminal a través del tiempo desensibilice a los jóvenes sobre las consecuencias de su conducta en ellos mismos y en los otros, posibilitando que sus rasgos de dureza personal sean más estables (Frick, et al., 2003).

Finalmente, los autores señalan que aunque este grupo de estudios cuenta con abundantes limitaciones, debidas principalmente al pequeño tamaño de los grupos en los que se efectuaron los seguimientos, sus resultados sugieren que los rasgos de D/I permiten identificar a un grupo de jóvenes en alto riesgo de mantener conductas antisociales en su desarrollo hacia la adultez. Sin embargo, los resultados no entregan una estimación de cuántos de estos jóvenes reunirán los criterios diagnósticos establecidos para la psicopatía en la adultez (Frick, et al., 2003; Frick et al., 2005); lo que sí es claro, es que estos jóvenes ameritan recibir estrategias de intervención diferenciadas con respecto a las recibidas por los otros jóvenes con problemas conductuales (Frick et al., 2005).

\section{Prevención y tratamiento}

El mayor debate y controversia que ha suscitado el estudio de la psicopatía infanto-juvenil se debe, principalmente, a la visión fatalista del concepto que asume la imposibilidad 
de intervención. Sin embargo, la idea de que la psicopatía infanto-juvenil es una condición intratable es cuestionada por la practica clínica de psicólogos infanto-juveniles que reportan que niños y adolescentes con características asociadas a psicopatía presentan ganancias moderadas cuando son tratado en psicoterapia (Salekin, 2002; Salekin $\&$ Frick, 2005). Además, estudios sobre el desarrollo de la personalidad muestran que los rasgos son más maleables en las primeras etapas de la vida que en la adultez (Roberts \& DelVecchio, 2000) y hay evidencia de posibilidades de cambio en jóvenes con rasgos psicopáticos (Vitacco, Neumann, Robertson \& Durrant, 2002).

Así como la detección precoz de la psicopatía infanto-juvenil y el conocimiento de las características de los jóvenes en riesgo de psicopatía está bien respaldada en la literatura, no sucede lo mismo con las estrategias de tratamiento para los jóvenes psicópatas. Aunque se ha intentado proveer a los especialistas de algunas bases o directrices para actividades preventivas e intervenciones terapéuticas tempranas, la información se encuentra dispersa en diferentes fuentes que tratan indirectamente el tema.

Una revisión de intervenciones terapéuticas con psicópatas realizada por Salekin (2002) señala algunos aspectos que los psicólogos y profesionales encargados del tratamiento de jóvenes con rasgos de psicopatía infanto-juvenil deberían considerar. Entre ellos están los siguientes:

Los métodos de tratamiento empleados con personas con características psicopáticas cubren un amplio rango que incluye desde tratamientos electroconvulsivos al psicodrama y el psicoanálisis tradicional. Los métodos de tratamiento generalmente se vinculan con una concepción de psicopatía como predisposición temperamental única-como es el caso de las formulaciones de Frick y Ellis (1999) y de Lynam $(1996,1997)$ revisadas en este artículo-, o sugieren que ésta se desarrolla a partir de un conjunto específico de condiciones ambientales (e.g. problemas de apego por maltrato). Sin embargo, se debe considerar que la psicopatía se puede desarrollar de diferentes formas en diferentes personas: a partir de predisposiciones, de ambientes difíciles, $\mathrm{y}$ también a partir de la interacción temperamento-ambiente. En todos los casos, aun en los modelos temperamentales, se debe tener en cuenta que la interacción con los padres, los pares y la sociedad pueden propiciar tanto desvinculación y agresión como apego y conductas prosociales en los jóvenes.

Respecto de los métodos de tratamiento, la revisión de Salekin (2002) muestra, sorpresivamente, que la terapia psicoanalítica parece ser efectiva en el tratamiento de la psicopatía y que sólo es superada levemente por tratamientos cognitivo-conductuales (59\% v/s 62\%). Los resultados indican que las terapias orientadas al insight ayudan a superar rasgos psicopáticos en la medida en que las personas aumentan su darse cuenta respecto de su estilo de vida; por su parte, las terapias cognitivo-conductuales parecen ser efectivas porque abordan los pensamientos del individuo sobre sí mismo, los otros y la sociedad. En ambos casos se trabaja directamente con los rasgos psicopáticos. Cabe señalar, que terapias eclécticas, que integran técnicas cognitivo-conductuales con técnicas de insight aumentan su efectividad al $86 \%$.

Salekin (2002) también señala que las comunidades terapéuticas fueron el método menos efectivo (25\%). A juicio del autor, este resultado no es sorpresivo porque aunque las comunidades terapéuticas frecuentemente reciben jóvenes con características psicopáticas, los programas de tratamiento no están diseñados para tratar específicamente la sintomatología psicopática. Es más, pareciera que el escaso contacto psicólogo-paciente que suele haber en las comunidades terapéuticas tiene un impacto directo en la baja efectividad de estos programas. Respaldando esta idea, se señala que un programa de tratamiento que usó la modalidad de comunidad terapéutica, pero que fue diseñado específicamente para jóvenes con características psicopáticas (Ingram, Gerard, Quay \& Levison, 1970), tuvo una alta tasa de éxito (88\%); este programa se basó en el modelo de búsqueda de sensaciones y procuró mantener el interés de los jóvenes en el tratamiento, además la selección del equipo terapéutico consideró su entrenamiento específico y sus habilidades para trabajar con jóvenes psicópatas.

Otros aspectos que destaca la revisión de Salekin (2002) son que la proporción de jóvenes que se beneficia del tratamiento es sustancialmente mayor que la proporción de adultos (.96 v/s .63); que las intervenciones más exitosas implican tratamientos intensivos, complejos y elaborados, que incluyen psicoterapia individual, tratamiento con los miembros de la familia y trabajo grupal en conjunto con otros pacientes; además se precisa que el promedio de terapia individual requerido es de cuatro sesiones por semana por al menos un año.

Finalmente, Salekin (2002) señala que la prevención requiere de mayor estudio. Dado que las teorías del desarrollo sugieren que hay una predisposición o estilo temperamental en los orígenes de psicopatía infanto-juvenil, las intervenciones tempranas que toman en consideración estilos parentales competentes pueden ayudar a socializar positivamente a los niños; esos estilos parentales ameritarían ser desarrollados intencionadamente, sin embargo se debe tener en cuenta que requieren de un acompanamiento y supervisión intensivos con las familias.

\section{Discusión}

Esta revisión de la literatura ha tratado aspectos asociados con la definición y extensión del constructo de psicopatía infanto-juvenil, los instrumentos de evaluación actualmente en uso a nivel internacional, sus factores de riesgo y las perspectivas sobre tratamiento y prevención. Estos temas serán abordados reflexivamente a través de esta discusión. 
Con respecto a la definición del constructo, se cuestiona, siguiendo a Farrington (2005), la definición de Hare (2003) por el hecho de incluir en sí misma las conductas trasgresoras y antisociales que pretende explicar, encontrándose más apropiada para el trabajo con niños y jóvenes la propuesta de Cooke y Michie (2001) que no considera la conducta antisocial propiamente tal en la definición. Esta última definición evita la posible estigmatización de etiquetar a niños y jóvenes como "psicópatas" por el sólo hecho de presentar conductas antisociales; además, su conceptualización parece ser más relevante para estudiar la psicopatía en muestras de población general donde la trasgresión social y las conductas antisociales no son tan evidentes (Frick et al., 2000). Por otro lado, se hace evidente que la psicopatía infanto-juvenil incluye rasgos específicos de funcionamiento personal que están agrupados en la dimensión Dureza/ Insensibilidad propuesta por Frick et al. (1994). Se plantea que, posiblemente, en esta dimensión radica la esencia de la psicopatía y que amerita ser estudiada específicamente para poder desarrollar planes de intervención efectivos.

Con respecto a los instrumentos de evaluación se observa una gran proliferación de medidas, muchos estudios sobre sus características psicométricas e intentos de precisar mejor el constructo a través de su medición en diversos grupos y con diferentes herramientas. Se constata que, lamentablemente, esto no acontece en igual medida en Chile ni en otros países latinoamericanos donde los desarrollos son todavía insuficientes. Se destacan, como estudios promisorios, la evaluación general que se puede desarrollar a través del MACI (Alarcón, et al., 2005), el uso exploratorio de las escalas específicas de psicopatía derivadas de este instrumento (Alarcón, et al., 2009), y la reciente caracterización psicométrica del PCL:YV en adolescentes judicializados chilenos (Zuñiga, 2009). Además, se plantea que esta línea de trabajo es muy necesaria para proveer instrumentos válidos para nuestro contexto sociocultural; en este sentido es muy valioso el trabajo conjunto de grupos de investigadores y de equipos profesionales de las instituciones encargadas del cuidado y rehabilitación de los jóvenes infractores de ley.

En relación a prevención y tratamiento, se observa que no hay todavía estrategias que estén consolidadas y respaldadas empíricamente. En este ámbito pareciera que lo urgente, es decir, disminuir la conducta delictiva y bajar la tasa de reincidencia, es lo más importante; sin embargo, se observa que los programas de tratamiento más aceptados tienen objetivos centrados en lo conductual, muchas veces a través de medidas coercitivas, y que prestan poca atención al trabajo de las variables de personalidad que parecen constituir lo central de la psicopatía, particularmente en las poblaciones infantiles y juveniles donde recién se inicia la escalada delictual. Los tratamientos focalizados en las variables de personalidad plantean un gran desafío porque son, de acuerdo a Salekin (2002), muy complejos, de larga duración, altamente demandantes, y además requieren de profesionales especializados de alto nivel.

El abordaje de la psicopatía infanto-juvenil, tal como ha sido conceptualizada en este artículo, plantea grandes desafíos a los estudiosos, investigadores y profesionales a cargo de los niños y jóvenes involucrados. Se requiere de ellos flexibilidad y apertura con respecto al tema; trabajo sistemático, respaldado científicamente, en estrategias de diagnóstico y de intervención y, sobre todo, alta perseverancia pues, dados los antecedentes expuestos, pareciera que el avance rápido en esta área es tremendamente necesario pero muy difícil de lograr.

\section{Referencias}

Alarcón, P., Vinet, E., \& Salvo, S. (2005). Estilos de personalidad y desadaptación social durante la adolescencia. Psykhe, 14, 3-16.

Alarcón, P., Vinet, E., Salvo, S., \& Perez-Luco, R. (2009). Caracterización y evaluación multidimensional de adolescentes con desadaptación social. Informe Final. Proyecto FONDECYT 1070397, CONICYT, Gobierno de Chile.

Andershed, H., Gustafson, S., Kerr, M., \& Stattin, H. (2002). The usefulness of self-reported psychopathy-like traits in the study of antisocial behaviour among non-referred adolescents. European Journal of Personality, 16, 383-402.

Andershed, H., Kerr, M., Stattin, H., \& Levander, S. (2002). Psychopathic traits in non-referred youths: A new assessment tool. En E. Blauuw y L. Sheridan, (Eds.), Psychopaths: Current internacional perspectives (pp. 131-158). The Hague, Netherlands: Elsevier.

Campbell, M. A., Porter, S., \& Santor, D. (2004). Psychopathic traits in adolescent offenders: An evaluation of criminal history, clinical, and psychosocial correlates. Behavioral Sciences and the Law, 22, 23-47.

Cleckley, H. (1941). The mask of sanity. St Louis, MO: Mosby.

Cooke, D.J. \& Michie, C. (2001). Refining the construct of psychopathy: Towards a hierarchical model. Psychological Assessment, 13, 171188

Corrado, R. R., Vincent, G. M., Hart, S. D., \& Cohen, I. M. (2004). Predictive validity of the psychopathy checklist: Youth version for general and violent recidivism. Behavioral Sciences and the Law, 22, 5-22.

Dadds, M. R., Fraser, J., Frost, A., \& Hawes, D. J. (2005). Disentangling the underlying dimensions of psychopathy and conduct problems in chilhdood: A community study. Journal of Consulting and Clinical Psychology, 73, 400-410.

Essau, C., Sasagawa, S., \& Frick, P. J. (2006). Callous-Unemotional traits in a community sample of adolescents. Assessment, 20,10, 1-16.

Farrington, D. P. (2005). The importance of child and adolescent psychopathy. Journal of Abnormal Child Psychology, 33, 4, 489-497.

Forth, A. E., \& Burke, H. C. (1998). Psychopathy in adolescence: Assessment, violence, and developmental precursors. En D. J. Cooke, A. E. Forth y R. D. Hare, (Eds.). Psychopathy: Theory, research and implications for society (pp. 205-230). Londres: Kluwer.

Forth, A. E., Kosson, D. S., \& Hare, R. D. (2003). Psychopathy ChecklistYouth Version: Technical Manual. Toronto: Multi-Health Systems.

Frick, P.J. (2003). The Inventory of Callous-Unemotional Traits. Escala no publicada, Universidad de New Orleans.

Frick, P. J., Barry, C. T., \& Bodin, S. D. (2000). Applying the concept of psychopathy to children: Implications for the assessment of antisocial youth. En C. B. Gacono (Ed.), The clinical and forensic assessment of psychopathy: A practitioner's guide (pp. 3-24). Mahwah, NJ: Erlbaum.

Frick, P. J., Bodin, S. D., \& Barry, C. T. (2000). Psychopathic traits and conduct problems in community and clinic referred samples of children: Further development of the psychopathy screening device. Psychological Assessment, 13, 382-393. 
Frick, P. J., Cornell, A. H., Barry, C. T., Bodin, S. D., \& Dane, H. A. (2003) Callous-unemotional traits and conduct problems in the prediction of conduct problem severity, aggression, and self-report of delinquency. Journal of Abnormal Child Psychology, 31, 457-470.

Frick, P. J., Cornell, A.H., Bodin, S. D., Dane, H. A., Barry, C.T., \& Loney, B. R. (2003). Callous-unemotional traits and developmental pathways to severe conduct problems. Developmental Psychology, 39, 246-260.

Frick, P.J. \& Ellis, M. (1999). Callous-unemotional traits and subtypes of conduct disorder. Clinical child and Family Psychology Review, 2, 149-168.

Frick, P. J., \& Hare, R. D. (2001). The Antisocial Process Screening Device. Toronto: Multi-Health Systems.

Frick, P. J., Kimonis, E. R., Dandreaux, D. M., \& Farell, J. M. (2003). The 4 year stability of psychopathic traits in nonreferred youth. Behavioral Sciences and the Law, 21, 713-736.

Frick, P. J., O’Brien, B. S., Wootton, J. M., \& McBurnett, K. (1994). Psychopathy and conduct problems in children. Journal of Abnormal Psychology, 103, 700-707.

Frick, P. J., Stickle, T. R., Dandreaux, D. M., Farrell, J. M., \& Kimonis, E. R. (2005). Callous-unemotional traits in predicting the severity and stability of conduct problems and delinquency. Journal of Abnormal Child Psychology, 33, 471-487.

Green, K. W. (2005). Screening for psychopathy in youth: An investigation of three measures. Tesis doctoral, Doctor of Philosophy, Case Western Reserve University. Recuperado en marzo19,2009disponibleen http:// www.ohiolink.edu/etd/view.cgi?acc num=case1112555102

Hare, R.D. (1991). The Psychopathy Checklist-Revised. Toronto: MultiHealth Systems.

Hare, R.D. (2003). Psychopathy Checklist Revised (PCL-R) Toronto: Multi Health Systems.

Ingram, G. L., Gerard, R. E., Quay, H. C., \& Levison, R. B. (1970). An experimental program for the psychopathic delinquent: looking in the "correctional wastebasket". Journal of Research in Crime and Delinquency , 7, 24-30.

Karpman, B. (1949). The psychopathic delinquent child. American Journal of Orthopsychiatry, 20, 223-265.

Karpman, B. (1950). Psychopathic behavior in infants and children: A critical survey of the existing concepts. American Journal of Orthopsychiatry, 21, 223-272.

Lang, S., Klinteberg, B., \& Alm, P.O. (2002). Adult psychopathy and violent behavior in males with early neglect and abuse. Acta Psychiatrica Scandinavica, 106, 93-100.

Lynam, D. R. (1996). The early identification of chronic offenders: Who is the fledgling psychopath? Psychological Bulletin, 120, 209-234.

Lynam, D. R. (1997). Pursuing the psychopath: Capturing the fledgling psychopath in a nomological net. Journal of Abnormal Psychology, 106, 425-438

Marshall, L. A., \& Cooke, D. J. (1999). The childhood experiences of psychopaths: A retrospective study of familial and social factors. Journal of Personality Disorders, 13, 211-225.

Millon, T. (1993). Millon Adolescent Clinical Inventory Manual. Minneapolis, MN: National Computer Systems.

Moffitt, T. E. (1993). Adolescence-limited and life-course persistent antisocial behavior: A developmental taxonomy. Psychological Review, 100, 674-701.

Muñoz, L., \& Frick P. (2007). The reliability, stability, and predictive utility of the self-report version of the Antisocial Process Screening Device. Scandinavian Journal of Psychology, 48, 299-312.
Murrie, D. C., \& Cornell, D. G. (2000). The millon adolescent clinical inventory and psychopathy. Journal of Personality Assessment, 75 , 1, 110-125.

Organización Mundial de la Salud. (1992). CIE 10 / Clasificación Internacional de Enfermedades. Recuperado en abril 30, 2009 disponible en http://www.biblioteca.anm.edu.ar/icd.htm.

Pardini, D. A., \& Loeber, R. (2007). Interpersonal and affective features of psychopathy in children and adolescents: Advancing a developmental perspective. Introduction to special section. Journal of Clinical Child and Adolescent Psychology, 36, 269-275.

Paris, J. (2003). A biospychosocial model of psychopathy. En T. Millon, E. Simonsen, M. Birket-Smith, y R. Davis, (Eds.), Psychopathy: Antisocial criminal and violent behaviour (pp. 277-287). New York: Guilford Press.

Roberts, A. R., \& Bender, K. (2006). Overcoming Sisyphus: Effective prediction of mental health disorders and recidivism among delinquents. Federal Probation, 70, 2. Recuperado en marzo 2, 2009 disponible en http://www.uscourts.gov/fedprob/September_2006/sisyphus.html.

Roberts, B., \& DelVecchio, W. (2000). The rank order consistency of personality traits from childhood to old age: A quantitative review of longitudinal studies. Psychological Bulletin, 26, 3- 25.

Romero, E. (2001). El constructo psicopatía en la infancia y la adolescencia: Del trastorno de conducta a la personalidad antisocial. Anuario de Psicología, 32, 25-49.

Romero, E., Luengo, M., Gómez-Fraguela, J., Sobral, J., \& Villar, P. (2005). Evaluación de la psicopatía infanto juvenil: Estudio en una muestra de niños institucionalizados Anuario de Psicología Jurídica, 15, 23-40.

Salekin, R. T. (2002). Psychopathy and therapeutic pessimism: Clinical lore or clinical reality? Clinical Psychology Review, 22, 79-112.

Salekin, R. T., \& Frick, P. J. (2005). Psychopathy in children and adolescents: The need for a developmental perspective. Journal of Abnormal Clinical Psychology, 4, 403-419.

Salekin, R. T., Ziegler, T., Larrea, M., Anthony, V., \& Bennett, A. (2003). Predicting dangerousness with two Millon adolescent clinical inventory psychopathy scales: the importance of egocentric and callous traits. Journal of Personality Assessment, 80, 154-163.

Saltaris, C. (2002). Psychopathy in juvenile offenders: Can temperament and attachment be considered as robust developmental precursors? Clinical Psychology Review, 22, 729-752.

Spain, S. E., Douglas, K. S., Poythress, N. G., \& Epstein, M. (2004). The relationship between psychopathic features, violence, and treatment outcome: The comparison of three youth measures of psychopathic features. Behavioral Sciences and the Law, 22, 85-102.

Vitacco, M. J., Neumann, C. S., Robertson, A. A., \& Durrant, S. L. (2002). Contributions of impulsivity and callousness in the assessment of adjudicated adolescent males: A prospective study. Journal of Personality Assessment, 78, 98- 103.

Weiler, B. L., \& Widom, C. S. (1996). Psychopathy and violent behaviour in abused and neglected young adults. Criminal Behavior and Mental Health, 6, 253-271.

Werth, F., \& Sepúlveda, M. (2003) Delincuencia Juvenil en Chile: Tendencias y Desafios. Documento presentado en el seminario Gobierno local y Prevención en seguridad ciudadana. Santiago de Chile: Paz Ciudadana.

Zúñiga, D. (2009). Caracterización psicométrica del instrumento Psychopathy Checklist: Youth Version (PCL:YV). Tesis de Magíster en Psicología Jurídica y Forense, Universidad de La Frontera, Temuco, Chile. 